\title{
The Study of the Structural Colouration Observed in the Papilio crino Fabricius, 1792 Wings \\ Sackey $\mathrm{J}^{1,2 *}$, Nuru $Z Y^{1,2}$, Dompreh $\mathrm{KA}^{3}$ and Maaza $\mathbf{M}^{1,2}$ \\ ${ }^{1}$ UNESCO-UNISA Africa Chair in Nanosciences/Nanotechnology, College of Graduate Studies, University of South Africa (UNISA), Muckleneuk ridge, P.O. Box 392, \\ Pretoria, South Africa \\ ${ }^{2}$ Nanosciences African Network (NANOAFNET), iThemba Labs-National Research Foundation, Old Faure road, 7129, Somerset West, South Africa \\ ${ }^{3}$ Department of Physics, University of Cape Coast, Ghana
}

\begin{abstract}
The research on nano-photonics has been on the increase recently. Among the materials that exhibit stunning optical properties are the nano-photonic materials found in nature such as those on butterfly wings. The wing scales of papilio crino was analysed using the Scanning Electron Microscopy (SEM), UV-Vis-NIR spectrometer, and Optical Microscopy (OPM). Under the OPM, the wing scales was imaged both in normal and polarized $\left(45^{\circ}\right)$ light. The SEM images reveal concave surfaces separated by repeating pattern of ridges and cross ribs. The variation in colour across the concave surface modulation was evident from the optical microscopy images. Through the numerical simulation and CIELab colour space, it was shown that the green colour seen on the wings is a combination of yellow and blue light reflected from different microscopic regions on the wing concave surface. This shows application in the bio-mimicks of retroflection sensors for the detection and classification of optical systems.
\end{abstract}

Keywords: Papilio crino; Structures; Reflectance; Polarization; Concavity

\section{Introduction}

Nature has invented photonic structures thousands of years ago. The replication of these natural photonic structures is useful for the creation of model systems to better understand structural colours. The colour producing structures such as diffraction gratings, multilayer reflectors, and photonic crystals [1] are found in beetles [2], fruits [3], marine polychaete worms and butterfly wings [4]. Recently, several attempts have been made to investigate and copy the intricate structures on the wing scales of butterflies. However, the wing scales present extremely complex but fancy architecture. Such architectures constitute a technological challenge if one wants to mimic them, in particularly the colour mixing effect displayed by the papilionidae. The wings scales of the papailioned consist of regular deformed multilayer structures that are made from alternating layers of air and cuticle and create intense structural colours. Generally, the wing is composed of millions of scales, which resembles gathered sacks consisting of lower and upper laminae [5]. The scale structure is made of cuticle containing chitin, which has a refractive index of 1.56 [6].

Several papilionid species such as $P$. blumei, P. palinurus and $P$. buddha, are known to have colour producing structures. Vukusic et al. [7] investigated the origin of the green coloration in the scales of the Indonesian male $P$. palinurus butterfly and discovered that the green colour is a combination of yellow and blue iridescence. In their analysis, they described in details the scale concavities on the optical response and plane polarization. In addition, they concluded that the dual colour arises from a modulation imposed on the multilayer; producing the blue component in a process they called orthogonal-surface retroreflection. Tada et al. [8] analysed the scale microstructure on the $P$. blumei and discovered that the scales exhibit nonplanar specular reflection as different colour for different angles is observed. According to their analysis, the ridges and the cross ribs create a repeating pattern in the thin-film structure that changes the angle of incidence of light locally and produces changes in the colour with changing view angle. Previous studies on this species also show that the concave cuticle air multilayer on the wings scales causes polarization rotation for blue light after double bounce from the concavity edge [9]. The Papilio crino is another species in the Papilionidae family found in Central and Southern India. This species is a conspicuously large size butterfly with beautiful bright green colours through the transverse wing with tail like backward extension of brownish and black colours. In this study, we show that the green colour perceived on the wings of Papilio crino is due to the combination of colours caused by multiple bounces within the concavities.

\section{Materials and Methods}

The wings of the butterfly Papilio crino was collected from the Butterfly World Tropical Gardens in Cape-Town, South Africa. The parts of the wing showing the green colour was cut and placed on a conductive basking and later transferred to the EM1 Tech (K950X) to be coated with gold palladium in order to prevent charging. Once coated, it was imaged with Carl Zeiss Auviga Scanning Electron Microscopy (SEM) operated at $5 \mathrm{keV}$. The sample was imaged under the optical microscopy in reflection for normal incident light with different objective lenses at different exposure times. For the polarization of the sample, a U-AN 360-3 Olympus japan analyser was used to polarize the Olympus BX-UCB light source at $45^{\circ}$. Reflectance spectra at different angles of light incidence $\left(45^{\circ}, 30^{\circ}\right)$ were obtained as follows; a reflection probe made of 6 illumination fibers around a single read fiber, which results in a $25^{\circ}$ full angle field of view was used for the reflectance measurement. The single fibre leg was connected to the spectrophotometer (Ava-Spec-2048L) for detection and the other end was connected to the halogen and deuterium sources (Avalight-DHS).

*Corresponding author: Juliet Sackey, UNESCO-UNISAAfrica Chair in Nanosciences/ Nanotechnology, College of Graduate Studies, University of South Africa (UNISA), Muckleneuk ridge, P.O. Box 392, Pretoria, South Africa, Tel: 27788866609; E-mail: juliyalley@gmail.com

Received June 22, 2016; Accepted July 05, 2016; Published July 12, 2016

Citation: Sackey J, Nuru ZY, Dompreh KA, Maaza M (2016) The Study of the Structural Colouration Observed in the Papilio crino Fabricius, 1792 Wings. J Nanomed Nanotechnol 7: 387. doi:10.4172/2157-7439.1000387

Copyright: ( 2016 Sackey J, et al. This is an open-access article distributed under the terms of the Creative Commons Attribution License, which permits unrestricted use, distribution, and reproduction in any medium, provided the original author and source are credited. 
The probe end was placed through the reflection holder and pointed to the reference material for measurement. The WS-1(Lambertian diffuser) was used as the reference material and the light was blocked when taking the dark measurement. The Lambertian diffuser (matte white in colour) has a reflectivity greater than $98 \%$ (250 nm-1500 $\mathrm{nm}$ ) and well suited to quantify the overwhelmingly reflection of this butterfly in the studied section. The light from the tip of the probe end was shine on the wing and the measurement recorded with the Avasoft 8 software. Due to instability in the data, the experiment was repeated several times by warming up the light source, taking frequent dark and reference measurements and setting the integration time. It should be noted that the reflectance probe technique used has limitations when measuring reflectance of scattering surfaces. Some of the scattered light will not be collected by the fibre and transmitted into the spectrometer. Therefore, unless the reflectance standard and the sample have the same proportion of diffusely to specularly reflected light, then there will be an error in the total reflectance measured from the sample.

\section{Results and Discussion}

\section{Morphological analysis}

Figure 1 shows the image of the papilio crino Fabricius (1792) on the part of the wing studied (a1). It's corresponding SEM image shows two distinct layers on the wing scales (b). The cover scale consists of ordered array of concavities separated by ridges and cross ribs while the ground scale is flat with toothed edge. At higher magnification, the distance of separation between adjacent ridges on the concavity (c) is estimated as $\sim 6.48 \mu \mathrm{m}$. Meanwhile the ground scales are composed of longitudinal ridges separated by cross ribs and decorated with nanometre sized air chambers (d). The Fourier transform images clearly reveal the ridges (e) with periodicity $\sim 0.154 \mu \mathrm{m}^{-1}$.

As well established, the iridescence on the wings of butterfly is due to interference, scattering and diffraction caused by the multilayers. Against this background, the distance of separation between adjacent ridges on the concavities was measured. Due to the large value, the ridges on this species are unlikely to cause any significant diffraction. A similar observation was seen on the Papilio blumei [8]

Below the round concavity, a curved multilayer consisting of about 8 alternate layers of cuticle and air is observed in Figure 2a. The general arrangement on the wing scales on this species is similar to those on Urania, Prepona [10], the Indonesian male Papilio palinurus butterfly [11] and also on the Indonesian butterfly Papilio blumei [12].

Figure 3 shows the optical microscopy images in reflection for normal incident light (a) and in polarized light (b and c) on the parts of the wing labelled as a1. These images reveal the variation in colour across the concave surface modulation. The scales (center) in reflection for normal incidnet light appear yellowish-green (a). The centers of the scales is linked to the flat regions between the concavities as illusratred by Vukusic et al. [11]. When the light is crossed polarized, the yellowishgreen reflected light is extinguished, and we observe rather blue scales at the edges (b and c). That implys that the blue reflected light has undergone polarization conversion. The effect of the blue component is caused by pair of orthogonal multilayer surfaces that lies on opposite sides of each concavity [11] as observed on the SEM images. When the wing is placed between the polarizers, the reflection of light off the center of the cavities is suppressed while the retro-reflected light from four segments of the cavity edge is detected [12].

\section{Optical analysis}

Light incident off the centre into the concave multilayer surface induces multiple reflections within the concavity. In Figure 4, we observed reflectance spectra for the parts of the wing labelled as a1 under various angles of light incidence. The cuticle multilayer shows reflectance peaks at $18 \%$ and $8 \%$ for the different angles. These peaks are attributed to the optical interference resulting from the interaction of the incident light with the air-chitin stacks. The maximum wavelength for the peak at $30^{\circ}$ angle of light incidence is at $\max =555 \mathrm{~nm}$. This $\max$ value agrees well with the theoretical predication of the wavelength value in the green-yellow spectra range $(550 \mathrm{~nm})$. The peak shifts from $570 \mathrm{~nm}$ to $540 \mathrm{~nm}$ when the light incidence at an angle of $45^{\circ}$.

\section{Numerical modelling}

The optical reflectance on the wings of the papilio crino was determined numerically based on a model developed by [8]. The model describes the butterfly's iridescence. However, the effect due to the scale concavities on the optical response and plane polarization has been described in details by Vukusic et al. [11]. The simulation considers the $2 \mathrm{D}$ photonic crystal (Figure 2b) whose structure parameters are determined from the SEM image shown. The extinction coefficient, the angle of incidence, the thickness of the layer and the air cavity, the number of layers and the refractive index of the film material are included in the calculation. The film stack of the papilio crino consists of alternating laminae and air layers. As reported by many authors [13], the butterfly wing is made of cuticle containing chitin, which has a refractive index of 1.56 . The refractive index of the air layer was determined by considering the weighted average of air and chitin indices;

$$
\mathrm{n}_{\text {effective }}(1 \mathrm{~F}) \mathrm{n}_{\text {air }} \mathrm{Fn}_{\text {chitin }}
$$

Where $\mathrm{F}$ is the filling factor. The dimensions of the air cavity and chitin are $\mathrm{D}=220 \mathrm{~nm}$ and $\mathrm{d}=110 \mathrm{~nm}$, respectively. Consequently, a $0.66 \%$ filing factor was obtained according to the equation described elsewhere [14]. The actual number of air-chitin layers on the butterfly wing is $\sim 8$, thus, the first eight (8) layers were used in the numerical calculation. In the model, the thicknesses of the chitin layers were estimated as $200 \mathrm{~nm}$. The same values of thickness were assumed for the air layer. The light propagation through the multilayer was described by the transfer matrix, which incorporate the TE and the TM waves [15].

The numerical reflectance spectrum is presented in Figure 5. The reflectivity curve for the 8 layers shift towards a shorter wavelength, with well-defined peaks centred at $\max =500 \mathrm{~nm}$. As can be observe, there is discrepancy between the experimental result and that of the model. The discrepancy could be attributed to several factors. Firstly, the refractive index of the organic material keeps varying. Secondly, the thickness of the air cavity was assumed to be equal to that of the chitin layer, which may differ.

\section{CIELab colour space}

Insects colour space is much larger and richer than those of the human eye. The insects have as many as five photoreceptors, as opposed to the trichromatic visual system in human [16]. The human eye averages a multicolour spectrum into a monochromatic colour. In this paper, the CIELab colour space is used to illustrate the colours perceived on the wings of the Papilio crino. CIE $L^{\star} a^{\star} b^{*}$ is one of the models that describe the entire range of colours the human eye can see. It is a three-dimensional colour space consisting of a black-white axis $\left(L^{*}\right)$, a red-green axis $\left(a^{*}\right)$ and a yellow-blue axis $\left(b^{*}\right) . L^{*}$ is the luminosity which ranges from 0 to 100 [17]. The features that describe the colour in a three-dimensional space are the quantities that specify 
Citation: Sackey J, Nuru ZY, Dompreh KA, Maaza M (2016) The Study of the Structural Colouration Observed in the Papilio crino Fabricius, 1792 Wings. J Nanomed Nanotechnol 7: 387. doi:10.4172/2157-7439.1000387
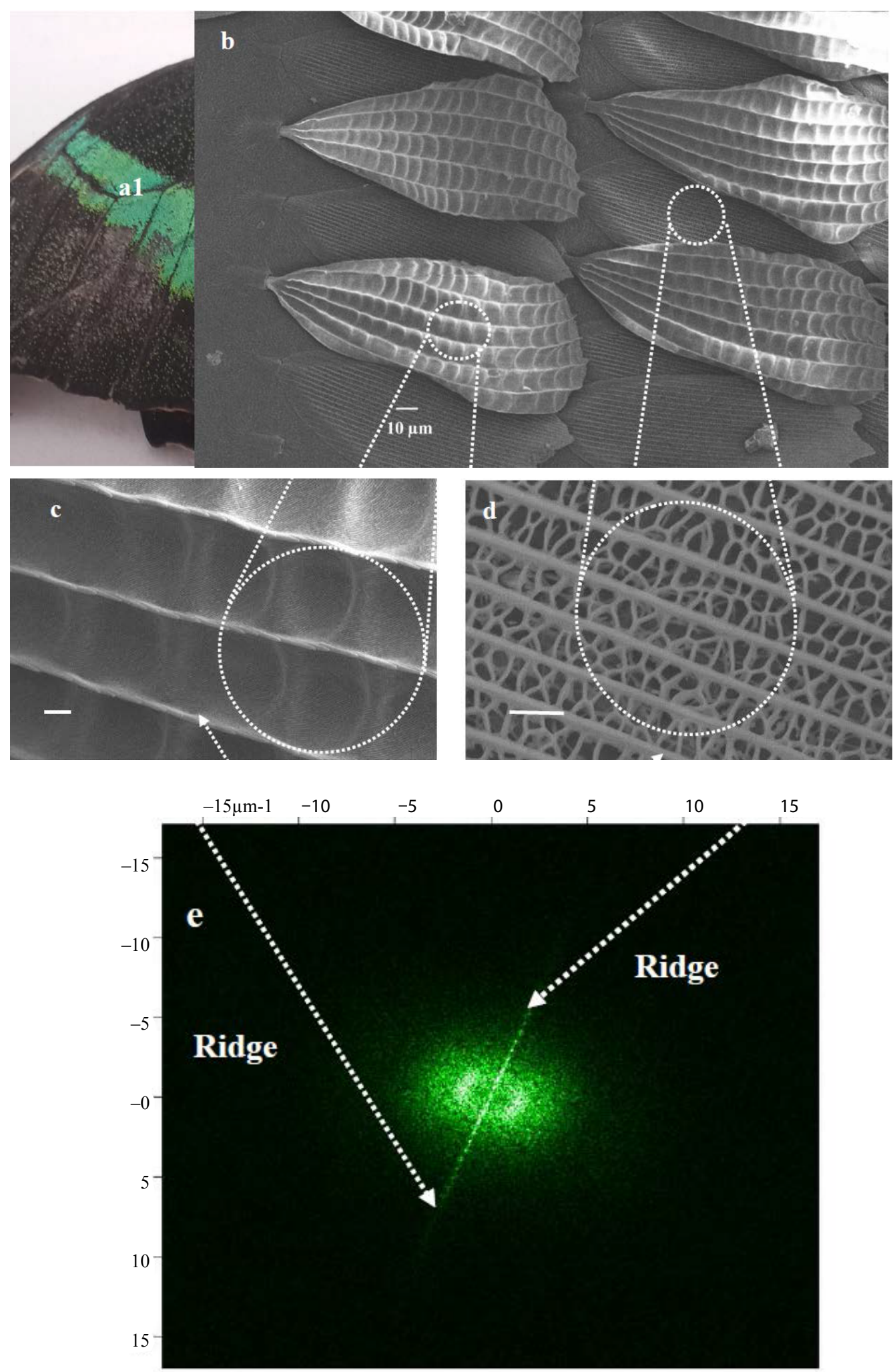

Figure 1: The image of the papilio crino Fabricius, 1792 wing showing the part studied (a1). The corresponding SEM images showing the two types of scales (b), the concavities on the surfaces (c), the surface of the ground scale showing the ridges and the air chambers (d), the Fourier transform image $(e)$. Scale bar: $(b)=10 \mu \mathrm{m}$, (c) $=1 \mu \mathrm{m}$ and $(\mathrm{d})=1 \mu \mathrm{m}$. 


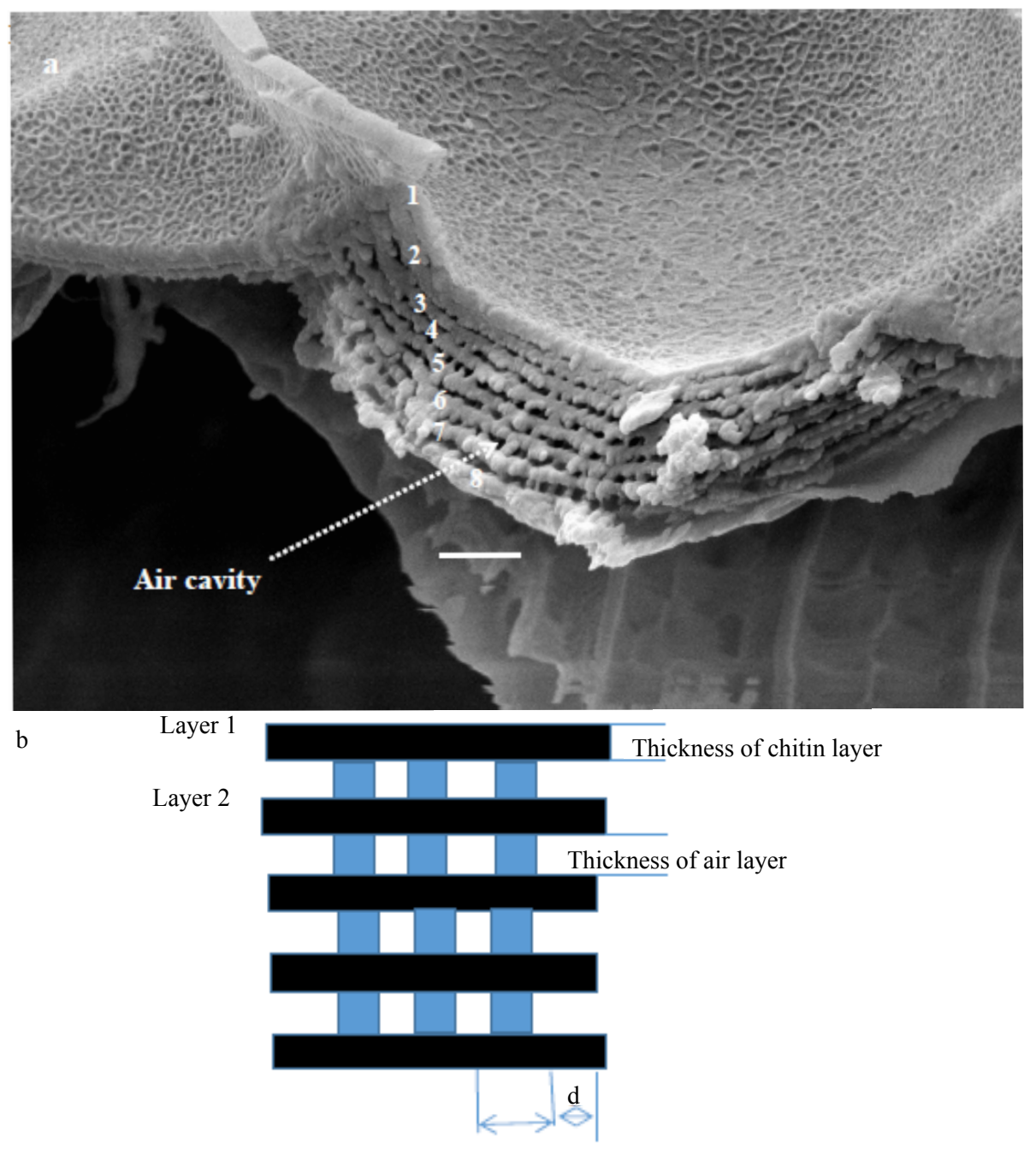

$\mathrm{D}$

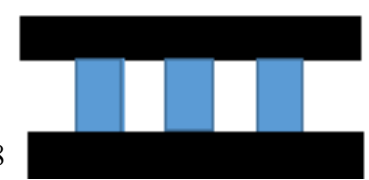

Figure 2: The cross section of the wing scale showing the multilayres (a), the $2 \mathrm{D}$ model of the papilio crino used for numerical simulation. Scale bar: $1 \mu \mathrm{m}$.

the colour, which are hue, brightness, and saturation. Hue is that quality that distinguishes one spectral colour from another. The hue of the colour is determined by $\mathrm{a}^{*}$ and $\mathrm{b}^{*} ; \mathrm{a}^{*}$ ranges from -128 (pure green) to +128 (pure red). Similarly $b^{*}$ ranges from -128 (pure blue) to +128 (pure yellow) [18]. The centre of this colour space is defined as the neutral point with $\mathrm{a}^{\star}$ and $\mathrm{b}^{\star}$ equal to zero.

Figure 6 presents the l.a.b colour space on the wings of the Papilio crino. The illuminate D65 is used with the k-means cluster algorithm in Matlab to define the colours. The results indicate that the colours perceived by the eye are actually a combination of colours being reflected off the microstructures on the scales.

\section{Conclusion}

The multilayer thin film structures that make up the wings of the papilio crino produce bright green colours from reflected light. As per the SEM images, the surfaces of the studied region comprise of ordered array of concavities. The flat regions between the concavities are linked to the centres of the scales, which appear yellowish-green in reflection for normal light as observed from the optical microscopy images. The edges of the scales however appear blue when the light is cross polarized. When white light is incident on this structure, yellow light hitting directly on the bottom of a concavity is selectively reflected, while blue light hitting the side of the concavity is reflected, hits again on the opposite side of the concavity wall, and is then reflected back in the reverse direction. A phenomena described by Vukusic et al. [11] as retro-reflection.

In addition, the cuticle multilayer on the concave surface shows reflectance peak at $\max =560 \mathrm{~nm}$, at the various angles of incidence. Further, we show through the numerical simulation on a $2 \mathrm{D}$ photonic crystal and CIELab colour space that the green colour seen on the wings is a juxtaposition of yellow and blue light reflected from different microscopic regions on the wing concave surface. 
Citation: Sackey J, Nuru ZY, Dompreh KA, Maaza M (2016) The Study of the Structural Colouration Observed in the Papilio crino Fabricius, 1792 Wings. J Nanomed Nanotechnol 7: 387. doi:10.4172/2157-7439.1000387
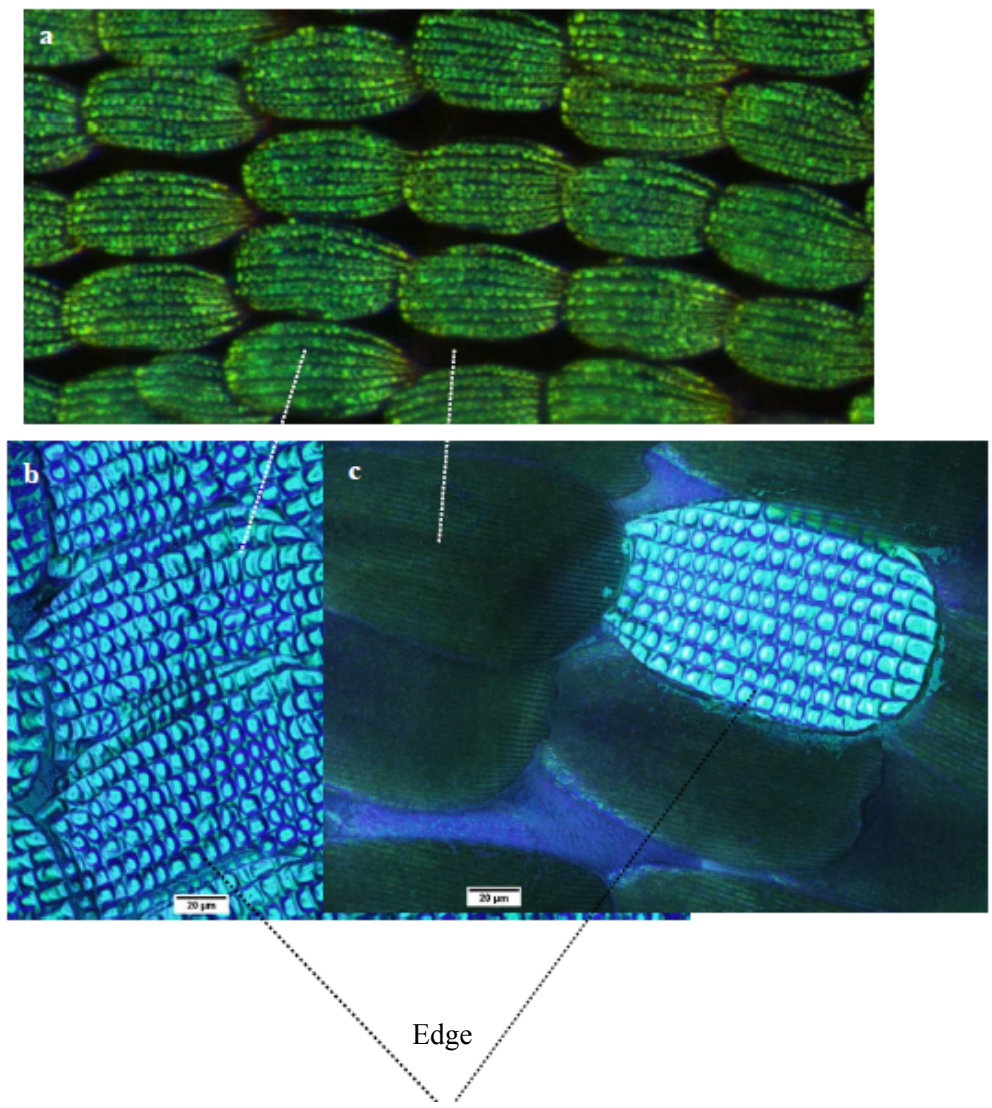

Figure 3: The optical microscopy images on the parts of the wing labelled as a1. The results show the yellowish-green unpolarised colours at centre of the scales when illuminated in reflection for normal incident light (a). The corresponding polarized images (b and c). At an angle of $45^{\circ}$, the reflected light is blue, highly polarized and goes out after double reflection in the direction of the incidence.

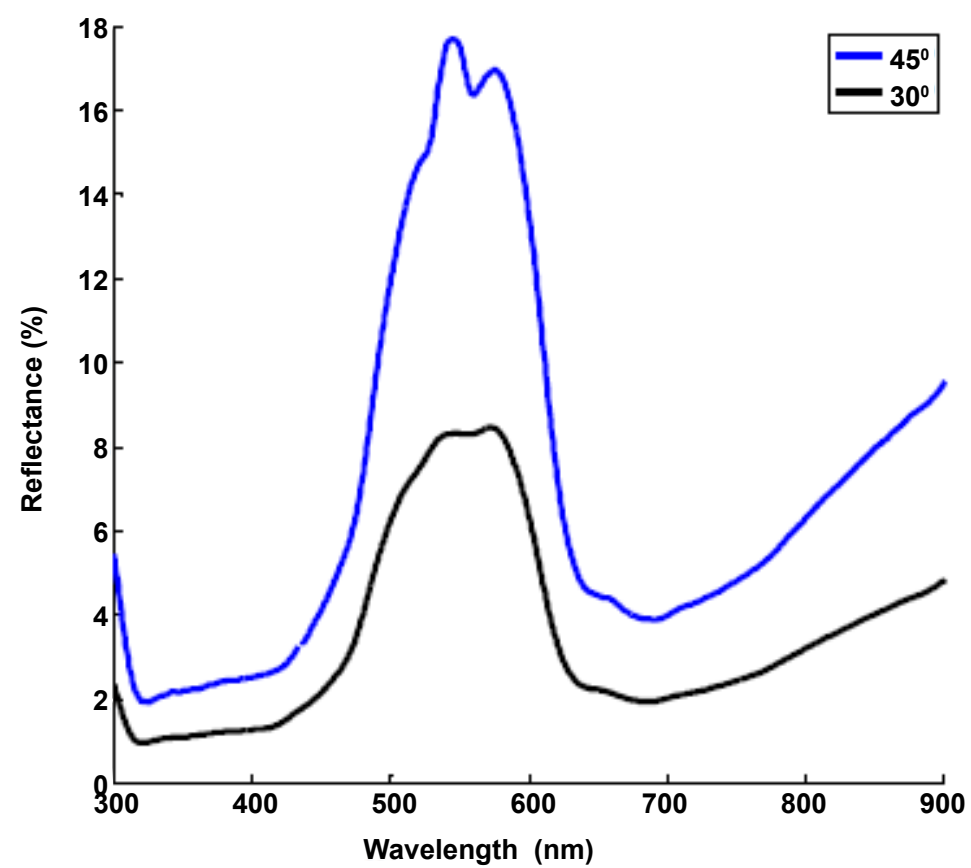

Figure 4: The reflectivity spectrum of the papilio crino under various angles of incidence on the parts of the wing labelled as a1. 
Citation: Sackey J, Nuru ZY, Dompreh KA, Maaza M (2016) The Study of the Structural Colouration Observed in the Papilio crino Fabricius, 1792 Wings. J Nanomed Nanotechnol 7: 387. doi:10.4172/2157-7439.1000387

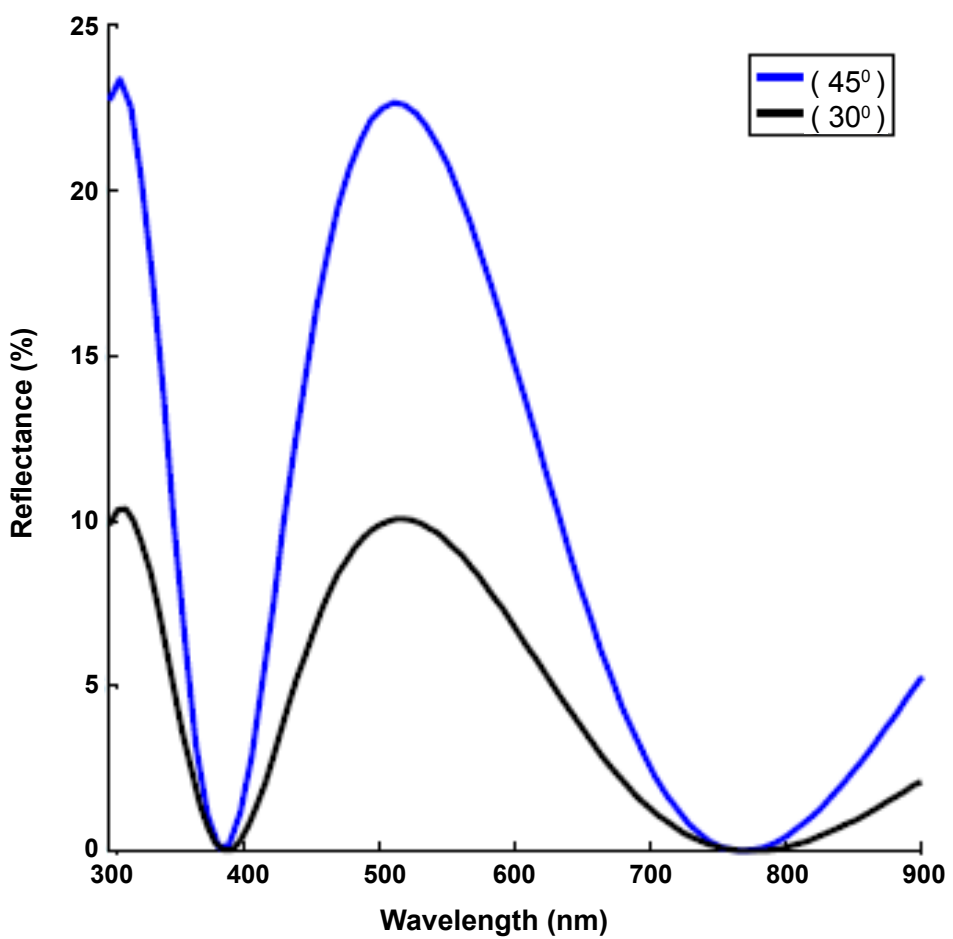

Figure 5: The calculated reflectivity spectrum for papilio crino at various angles of light incidence.

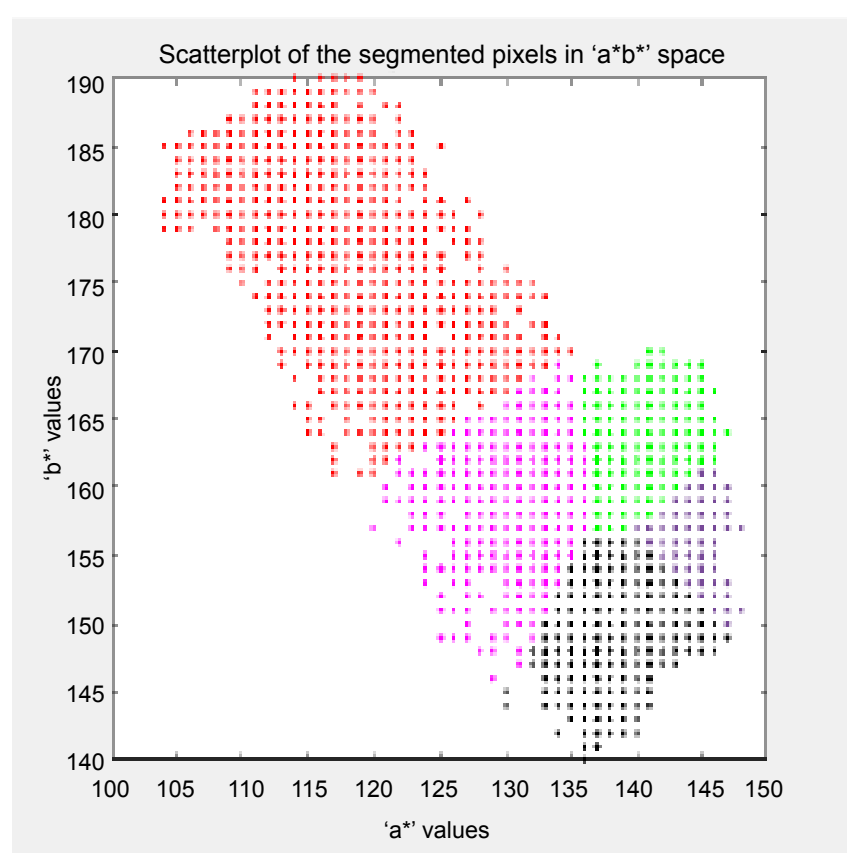

Figure 6: The I.a.b color space of the wing showing combination of different colours.

Although structural colour provides higher visibility than pigmentry, the purpose of this mechanism of colour generation is not clear. However, because of the multifunctionality of the butterfly wings, understanding the interaction of light with the wing scale would be an added advantage to develop better interference filters in the thin film industry.

\section{Acknowledgment}

This research program was generously supported by grants from the Organization of Women in Science for the Developing World (OWSDW) and the Abdus Salam ICTP, National Research Foundation of South Africa (NRF), iThemba LABS, the UNESCO-UNISA Africa Chair in Nanosciences and Nanotechnology, via the Nanosciences African Network (NANOAFNET) as well as the African Laser Centre (ALC) and Butterfly World Tropical Gardens to whom we are grateful.

\section{References}

1. Prum RO, Quinn T, Torres RH (2006) Anatomically diverse butterfly scales all produce structural colours by coherent scattering. J Exp Biol 209: 748-765.

2. Anderson T, Richards A (1942) An electron microscope study of some structural colours of insects. J Appl Phys 1: 748-758.

3. Lee DW (1991) Ultrastructural basis and function of iridescent blue colour of fruits in Elaeocarpus. Nature 349: 260-262.

4. Welch V, Vigneron J (2007) Beyond butterflies - the diversity of biological photonic crystals biological photonic crystals. Optical and Quantum Electronics 39: 295-300.

5. Miaoulis I, Wong P, Heilman B (1994) The effec tof micro scale and macroscale patterns on th eradiative heating of multilayerthin-filmstructures, in Micro scale Heat Transfe. ASME Heat Transfer Proceedings Series 29: 27-34.

6. Steindorfer MA, Schmidt V, Belegratis M, Stadlober B, Krenn JR (2012) Detailed simulation of structural color generation inspired by the Morpho butterfly. Opt Express 20: 21485-21494.

7. Vukusic P, Sambles JR, Lawrence CR (2000) Colour mixing in wing scales of a butterfly. Nature 404: 457.

8. Tada H, Mann S, Miaoulis I, Wong P (1999) Effects of a butterfly scale microstructure on the iridescent color observed at different angles. Opt Express 5: 87-92.

9. Kolle M, Salgard-Cunha PM, Scherer MR, Huang F, Vukusic P, et al. (2010) Mimicking the colourful wing scale structure of the Papilio blumei butterfly. Nat Nanotechnol 5: 511-515.

10. Berthier S (2007) Iridescences - The phyiscal colors of insects, New York Springer Science+Business Media 2007. 
Citation: Sackey J, Nuru ZY, Dompreh KA, Maaza M (2016) The Study of the Structural Colouration Observed in the Papilio crino Fabricius, 1792 Wings. J Nanomed Nanotechnol 7: 387. doi:10.4172/2157-7439.1000387

11. Vukusic P, Sambles JR, Lawrence CR (2000) Colour mixing in wing scales of a butterfly. Nature 404: 457.

12. Kolle M, Salgard-Cunha PM, Scherer MR, Huang F, Vukusic P, et al. (2010) Mimicking the colourful wing scale structure of the Papilio blumei butterfly. Nat Nanotechnol 5: 511-515.

13. Steindorfer MA, Schmidt V, Belegratis M, Stadlober B, Krenn JR (2012) Detailed simulation of structural color generation inspired by the Morpho butterfly. Opt Express 20: 21485-21494.

14. Gaillot P, Deparis O, Welch V, Wagner K, Vigneron P, et al. (2008) Composite organic-inorganic butterfly scales: Production of photonic structures with atomic layer deposition. Physical Review E 78: 0319221-0319226.
15. Stavenga DG, Wilts BD, Leertouwer HL, Hariyama T (2011) Polarized iridescence of the multilayered elytra of the Japanese jewel beetle, Chrysochroa fulgidissima. Philos Trans R Soc Lond B Biol Sci 366: 709-723.

16. Futahashi R, Kawahara-Miki R, Kinoshita M, Yoshitake K, Yajima S, et al. (2015) Extraordinary diversity of visual opsin genes in dragonflies. Proc Natl Acad Sci U S A 112: E1247-1256.

17. Medina J, Diaz J, Valero E, Nieves J, Vukusic $P$ (2014) Detailed experimental characterization of reflectance spectra of Sasakia charonda butterfly using multispectral optical imaging. Optical Engineering 5033111-0311115.

18. Luke SM, Vukusic P, Hallam B (2009) Measuring and modelling optica scattering and the colour quality of white pierid butterfly scales. Opt Express 17: $14729-14743$. 